\title{
De Novo DNA Synthesis in Aedes aegypti Midgut Cells as a Complementary Strategy to Limit Dengue Viral Replication
}

\author{
Javier Serrato-Salas, Salvador Hernández-Martínez, Jesús Martínez-Barnetche, \\ Renaud Condé, Alejandro Alvarado-Delgado, Federico Zumaya-Estrada and \\ Humberto Lanz-Mendoza*
}

Centro de Investigaciones Sobre Enfermedades Infecciosas, Instituto Nacional de Salud Pública, Cuernavaca, Mexico

\section{OPEN ACCESS}

Edited by:

Akio Adachi,

Tokushima University, Japan

Reviewed by:

Ramesh C. Dhiman,

National Institute of Malaria Research

(ICMR), India

Lalita Gupta,

Chaudhary Bansi Lal University, India

Tatsuo Shioda,

Osaka University, Japan

*Correspondence: Humberto Lanz-Mendoza

humberto@insp.mx

Specialty section: This article was submitted to

Virology,

a section of the journa

Frontiers in Microbiology

Received: 28 November 2017

Accepted: 10 April 2018

Published: 26 April 2018

Citation:

Serrato-Salas J, Hernández-Martínez $S$

Martínez-Barnetche J, Condé R,

Alvarado-Delgado $A$,

Zumaya-Estrada F and

Lanz-Mendoza H (2018) De Novo DNA Synthesis in Aedes aegypti Midgut Cells as a Complementary

Strategy to Limit Dengue Viral

Replication. Front. Microbiol. 9:801.

doi: 10.3389/fmicb.2018.00801
Aedes aegypti is the main vector of Dengue Virus, carrying the virus during the whole mosquito life post-infection. Few mosquito fitness costs have been associated to the virus infection, thereby allowing for a swift dissemination. In order to diminish the mosquito population, public health agency use persistent chemicals with environmental impact for disease control. Most countries barely use biological controls, if at all. With the purpose of developing novel Dengue control strategies, a detailed understanding of the unexplored virus-vector interactions is urgently needed. Damage induced (through tissue injury or bacterial invasion) DNA duplication (endoreplication) has been described in insects during epithelial cells renewal. Here, we delved into the mosquito midgut tissue ability to synthesize DNA de novo; postulating that Dengue virus infection could trigger a protective endoreplication mechanism in some mosquito cells. We hypothesized that the Aedes aegypti orthologue of the Drosophila melanogaster hindsight gene (not previously annotated in Aedes aegypti transcriptome/genome) is part of the Delta-Notch pathway. The activation of this transcriptional cascade leads to genomic DNA endoreplication. The amplification of the genomic copies of specific genes ultimately limits the viral spreading during infection. Conversely, inhibiting DNA synthesis capacity, hence endoreplication, leads to a higher viral replication.

Keywords: Aedes aegypti, DENV, DNA endoreplication, antiviral response, hindsight, delta, notch

\section{INTRODUCTION}

Vector borne virus infections have recently caught the public attention with the emergence of Zika (ZIKV) and Chikungunya (CHIKV) viruses in America. Simultaneously, a re-incidence of Dengue virus (DENV) infections has been observed. Dengue infection causes a disease that present the highest mortality and morbidity rates amongst the aforementioned viruses; being the sole life-threatening virus infection. In America, over the last few years these three emergent diseases constituted an important burden to the local public health care systems. Aedes aegypti is the main insect vector for DENV, CHIKV, and ZV (OMS, 2009).

When the mosquitoes ingest a virus-infected blood meal, the virus reaches the midgut, invades and escapes this barrier. The tracheal and/or muscles system may also act as a viral escape conduit into the hemolymph, allowing virus spread in virtually all organs in the insect. 
Infection persist in most mosquito tissues during the insect lifetime. Nevertheless, the amount of viral antigen and titers can decline over time (Romoser et al., 2004; Salazar et al., 2007).

In insects, effector mechanisms like phagocytosis, nodule encapsulation, melanization, and expression of reactive oxygen species; along with antimicrobial peptides; can target and kill microorganisms. Mosquitoes ability to limit viral spreading has been found to rely on the classical insect innate immune cascades. The Aedes aegypti immune response acts as a key regulator for acquiring, maintaining and transmitting virus; a phenomenon known as vectorial competence. Indeed, the Toll, IMD, Jak/STAT, and RNAi signaling cascades are activated in DENV presence (Salazar et al., 2007; Xi et al., 2008; Sánchez-Vargas et al., 2009; Souza-Neto et al., 2009; Sim and Dimopoulos, 2010; Raquin and Lambrechts, 2017).

However, DENV fend off these barriers and, as a consequence, are disseminated by the mosquitoes and spread to humans (Cheng et al., 2016). This virus do repress Aedes aegypti antimicrobial peptide production during its infection course (Souza-Neto et al., 2009). Indeed, during bacterial challenges, DENV-infected Aedes aegypti cells show lowered cecropin and defensin production, when compared with virus-uninfected cells (Sim and Dimopoulos, 2010).

There exists a delicate balance between restrictive and permissive factors. Viral particles invade tissues inducing the upregulation of the host permissive factors, then the virus titer reaches a peak, and later on the host restrictive factors diminish the viral propagation. This response is tightly regulated by immune pathways and some other factors not yet explored.

In order to design alternative and complementary strategies to fight arbovirus diseases, we need to gain a better understanding of the virus-vector molecular interactions. Several transcriptomics published works showed that, during virus infection, many cell cycle genes and DNA synthesis core components are differentially expressed in mosquito tissues. The authors attribute these genes differential expression to mitochondrial stress and metabolism dysregulation (Xi et al., 2008; Behura et al., 2011; Chauhan et al., 2012; Ramirez et al., 2012; Khoo et al., 2013; Tsujimoto et al., 2017). These genes have not been attributed to a physiological response against DENV nor to endoreplication of effector genes limiting viral spreading.

Many organisms can replicate their genomes without segregating chromosomes during their immature developmental stages. This phenomenon increases the DNA content in the cells, allowing higher transcription and swifter protein output. DNA replication devoid of mitosis enhance macromolecular secretion. This process is widespread in protists, plants and many animals including arthropods, mollusks, and mammals (Edgar and OrrWeaver, 2001; Lee et al., 2010).

In Drosophila melanogaster, this phenomenon has been well characterized during oocyte development. It enables the vitellogenesis and egg shell formation. Inside the egg chamber, the Delta-Notch interaction between follicle and nurse cells switched the cell cycle from mitosis to endocycle. The endocycle increases the number of transcription binding loci, thereby maximizing mRNA and protein synthesis in a tightly regulated process (Edgar and Orr-Weaver, 2001; Lee et al., 2010; Palmer et al., 2014).

In the fly adult midgut, the intestinal stem cells activate the Delta-Notch signaling in order to induce asymmetrical division to generate new stem cells and enterocytes subtypes (Guo and Ohlstein, 2015). Beside growth; stress, injury and bacteria killing are proposed to be other stimulus involved in midgut endoreplication cycles (Buchon et al., 2009; Fox and Duronio, 2013; Castagnola and Jurat-Fuentes, 2016).

In adult fly, pro-hemocytes differentiation to crystal cells is also mediated by hindsight (hnt) and involves Delta-Notch activation (Pickup et al., 2009; Terriente-Felix et al., 2013). hnt also functions as a regulating switch for cell differentiation in developmental and mature stages (Baechler et al., 2016).

Upon insult (puncture) Bombyx mori larval midgut cells endoreplicate their genomic DNA through asymmetric division without any apoptosis processes. Surrounding the wound site, the intestinal stem cells divide asymmetrically, and their enhanced DNA replication help repair damage. Cisplatin injection inhibited the post traumatic endoreplication process observed (Huang et al., 2016).

In Anopheles albimanus, pathogen challenges with Plasmodium parasite, yeast wall and dead gram-negative bacteria activate the immune system, triggering DNA endoreplication mechanism (Hernandez-Martinez et al., 2006; HernándezMartínez et al., 2013; Contreras-Garduño et al., 2015). Insofar, the molecules involved in this process have not been thoroughly unveiled.

In this paper, we describe a DNA endoreplication Aedes aegypti antiviral strategy against the DENV infection.

Some DNA sequences coding for homologs of the proteins involved in D. melanogaster endoreplication can be found in Aedes aegypti transcriptome/genome. Here, we explored the presence of the main Notch canonical pathway components in Aedes aegypti transcriptome/genome.

We hypothesized that Aedes aegypti female midgut cells could enter endoreplication cycles upon insult in a similar fashion as observed in D. melanogaster model. We postulate that endoreplication is triggered during DENV infection, and that endoreplication limits the viral propagation through enhanced transcription of immune effectors. We tracked 5-bromo-2deoxyuridine DNA incorporation to determine de novo DNA synthesis into the genome of adult mosquitoes cells. In this work, we also assess Notch transcriptional activity in mosquito midgut during DENV infection.

\section{MATERIALS AND METHODS}

\section{Mosquitoes Rearing}

Anopheles albimanus white-striped pupal phenotype strain (Chan et al., 1994) and Aedes aegypti Rockefeller strain were reared in the insectary of the INSP (Instituto Nacional de Salud Pública), 100 females Anopheles albimanus and Aedes aegypti mosquitoes per group were maintained under $60-80 \%$ of relative humidity, $28 \pm 1{ }^{\circ} \mathrm{C}$ of environmental temperature, and $12 / 12 \mathrm{~h}$ dark/light photoperiod cycles. Adult mosquitoes were fed ad libitum with cotton soaked in sucrose solution ( $8 \%$ for 
Anopheles albimanus and 10\% for Aedes aegypti) until blood feeding 3-5 days post-emergence. Mosquitoes were blood fed by placing an artificial glass feeder with a parafilm membrane. Mosquitoes were fed with heparinized rabbit blood for egg production. Mosquitoes were left in pans in insectary conditions until processed.

\section{Viral Propagation and Focus Forming Assays}

DENV serotype 2 New Guinea C strain was obtained from the INSP Dengue virus collection. DENV-2 passage/amplification were realized twice in suckling mice brain and thrice in $\mathrm{C6} / 36$ cells. Suckling mice infected were euthanized in a $\mathrm{CO}_{2}$ closed-chamber according to Mexican law "Ley de Regulación Federal para el Manejo y Experimentación de Animales de la Secretaria de Agricultura, Ganadería, Desarrollo Rural, Pesca y Alimentación (SAGARPA) en la Norma Oficial NOM-062-ZOO1999, Especificaciones técnicas para la producción, cuidado y uso de los animales de laboratorio", and INSP internal bioethical committee approval.

C6/36 cells (ATCC-CRL 1660) were grown at $28^{\circ} \mathrm{C}$ in $75 \mathrm{~cm}^{2}$ flasks using L-15 medium (Thermo Scientific) supplemented with $10 \%$ of heat-inactivated fetal bovine serum (FBS, Byproductos). Cell monolayers were prepared seeding scrapped cells into new flasks with fresh media.

The viral stock was obtained by infecting C6/36 cells with 0.1 MOI for 7 days. DENV-2 infected and mock-infected cells were lysed by three freeze/defreeze cycles, clarified by centrifugation at $15,000 \mathrm{~g}$ at $4^{\circ} \mathrm{C}$ during $1 \mathrm{~h}$, and titrated using a focus forming units assay mentioned below. Mock-infected samples consisted in C6/36 cultured in L-15 media, collected similarly (Morens et al., 1985).

LLC-MK $\mathrm{MK}_{2}$ cells (ATCC CCL-7) were propagated at $37^{\circ} \mathrm{C}$ in 25 $\mathrm{cm}^{2}$ flasks using MEM (Thermo Scientific) supplemented with $10 \%$ FBS growth media.

Viral titers were determined by Focus Forming Units Assay (FFUs). Media from the cell plates was removed and washed twice with PBS before adding $0.22 \mu \mathrm{M}$ membrane-filtered triplicate of $\log _{10}$ serial dilutions of the samples to be titrated. Dilutions were incubated $2 \mathrm{~h}$ at $37^{\circ} \mathrm{C}$, and then virus-dilutions were replaced by growth media. Plates were incubated 6 days at $37^{\circ} \mathrm{C}$ before removing the media. Cells were fixed overnight at $-20^{\circ} \mathrm{C}$ with methanol, then cells were rehydrated with phosphate buffer saline (PBS) pH $7.4(137 \mathrm{mM} \mathrm{NaCl}, 2.7 \mathrm{mM}$ $\mathrm{KCl}, 1.47 \mathrm{mM} \mathrm{KH} \mathrm{PO}_{4}, 8.1 \mathrm{mM} \mathrm{Na} \mathrm{HPO}_{4}$, all from Sigma). Endogenous peroxidase activity was blocked by adding hydrogen peroxide $0.3 \%$ to the PBS solution. Wells were coated $1 \mathrm{~h}$ at $37^{\circ} \mathrm{C}$ with a mouse antibody against Dengue M Protein $(2 \mathrm{H} 2$, a kindly gift of Dr. Juan Ludert, CINVESTAV, IPN) diluted 1:25 in growth media. The supernatant is then flushed and excedent media washed away. Wells are incubated $1 \mathrm{~h}$ at $37^{\circ} \mathrm{C}$ with goat anti-mouse-IgG-horseradish peroxidase (Abcam) diluted $1: 1,000$. DAB substrate (Thermo Scientific) is then used to reveal immunoassay. Focus were counted and titer was calculated using Reed-Muench formula described elsewhere (Baer and Kehn-Hall, 2014).

\section{Mosquitoes Oral Infection With DENV}

A suspension of rabbit red blood cells was prepared as follows, $10 \mathrm{~mL}$ of heparinized blood were centrifuged for $5 \mathrm{~min}$ at $600 \mathrm{~g}$, the cellular pellet was washed three times with PBS. The rabbit red blood cells were resuspended in the same volume of MEM media (Thermo Scientific). This suspension was supplemented in $50 \% / 50 \%$ proportion with $1 \times 10^{8} \mathrm{FFUs} / \mathrm{mL}$ of DENV-2 NGC. Mock control infections were prepared equally with uninfected C6/36 lysate.

Six of all the experiments were carried out using 3-5 days post-emergence Aedes aegypti females, with approximately 50200 individuals per group. After oral feeding, mosquitoes were cold anesthetized $\left(\sim 4^{\circ} \mathrm{C}\right)$ during 5-10 min. Engorged females were placed in a new pan and maintained until processed with sucrose cotton soaked as described earlier. Biohazard residues were disposed following bioethical standard procedures.

\section{Mosquitoes Midgut Dissection, RNA and DNA Extraction}

Mosquitoes were anesthetized on ice and their midguts were dissected in a drop of sterile PBS. The resulting tissues were put in Trizol reagent (Thermo Scientific) for RNA extraction at $-20^{\circ} \mathrm{C}$. Other midgut pools were placed in Phenol-Chloroform-Isoamyl Alcohol mixture for DNA extraction. For viral titration, pools of 5 or 10 midguts were put in $50 \mu \mathrm{L}$ of MEM and frozen at $-70^{\circ} \mathrm{C}$ until processing.

Midgut pools placed in Trizol (Invitrogen) were homogenized using a small piston until macerated. RNA was obtained according to manufacturer's instructions and used for RT-PCR assays as per protocol.

cDNA synthesis was carried out using RevertAid Premium Transcriptase kit (Thermo Scientific) following manufacturer's instructions. Samples were saved for DNA extraction following manufacturer's instructions. To eliminate RNA traces, samples were incubated with RNAse A (Gibco) for $1 \mathrm{~h}$ at $37^{\circ} \mathrm{C}$.

Quantitative PCR were performed using ViiA 7 Real-Time PCR System (Applied Biosystems) with the QuantStudio Real Time Software v1.3. Reactions were realized in a total volume of $10 \mu \mathrm{L}$ containing $5 \mu \mathrm{L}$ of SYBR Green PCR Master mix, 1.5 ng of cDNA template, $250 \mathrm{nmol}$ of each one of primers, and volume completed with nuclease-free water. Reaction conditions were the next: $50^{\circ} \mathrm{C}$ for $2 \mathrm{~min}, 95^{\circ} \mathrm{C}$ for $10 \mathrm{~min}$ followed by 40 cycles of denaturation at $95^{\circ} \mathrm{C}$ for $15 \mathrm{~s}$, annealing and extension at $60^{\circ} \mathrm{C}$ for $1 \mathrm{~min}$. The $\mathrm{Ct}$ value obtained from the tested gene relative to the reference gene, was used to obtain delta Ct values of infected/uninfected samples, as well cisplatin/nocisplatin treatment mentioned below. S7 gene (ribosomal unit S70) was elected as the reference gene based on previous lab published papers (Moreno-García et al., 2015; Vargas et al., 2016). Relative expression values were obtained using the delta-delta cycle threshold (DDCT) method (Bubner et al., 2004).

\section{DNA Synthesis De Novo in Mosquitoes}

Female Anopheles albimanus and Aedes aegypti mosquitoes were fed ad libitum with a cotton soaked in sucrose solution plus $100 \mu \mathrm{g} / \mathrm{ml}$ of BrdU. At day 3, mosquitoes were mock- and 
DENV-2 fed. Tissues samples were taken equal as kinetics infection assay mentioned above.

\section{Immunofluorescence Assay for BrdU Incorporation}

Full midgut and abdomen tissues were dissected and fixed $2 \mathrm{~h}$ with $4 \%$ paraformaldehyde (Sigma), fixative excess was removed from samples and permeabilized with methanol at $-20^{\circ} \mathrm{C}$ for $10 \mathrm{~min}$ and washed with PBS-Tween $1 \%$ (PBS$\mathrm{T}$ ), samples were hydrolyzed with $\mathrm{HCl} 2 \mathrm{~N} 1 \mathrm{~h}$ at $37^{\circ} \mathrm{C}$, and neutralized by three changes of Hank's solution $(\mathrm{NaCl} 137 \mathrm{mM}$, $\mathrm{KCl} 5.4 \mathrm{mM}, \mathrm{Na}_{2} \mathrm{HPO}_{4} 0.25 \mathrm{mM}, \mathrm{KH}_{2} \mathrm{PO}_{4} 0.44 \mathrm{mM}, \mathrm{CaCl} 2$ $1.3 \mathrm{mM}, \mathrm{MgSO}_{4} 1 \mathrm{mM}, \mathrm{NaHCO}_{3} 4.2 \mathrm{mM}$ ) for $10 \mathrm{~min}$ each and washed three times with PBS-T. Unspecific antibody binding was blocked $1 \mathrm{~h}$ at $37^{\circ} \mathrm{C}$ with bovine serum albumin in PBS (PBS-A). Samples were incubated overnight at $4^{\circ} \mathrm{C}$ with a FITC-labeled-mouse anti-BrdU monoclonal antibody (Roche). After time, samples were rinsed with PBS-T and mounted on slide using Vectashield (Vector Laboratories). Fluorescence was recorded using a photo-epifluorescente microscope (Nikon E600 ), protocol was followed as it reported (Hernandez-Martinez et al., 2006; Hernández-Martínez et al., 2013; Contreras-Garduño et al., 2015).

\section{ELISA Assay for BrdU Incorporation}

For ELISA assay DNA was extracted as mentioned above, midgut gDNA samples were re-suspended in $50 \mu \mathrm{L}$ of PBS. DNA concentration was determined measuring 230/260 nm absorbance using Nanodrop ${ }^{\odot}$ spectrophotometer. One to ten micrograms of DNA samples diluted in bicarbonate buffer $\mathrm{pH}$ 9.2 were transferred to a pre-treated poly-L-lysine 96 wells ELISA plate and incubated at $4^{\circ} \mathrm{C}$ overnight. The BrdU incorporated to the mosquito midguts cell's nucleus was determined by ELISA assay; using peroxidase conjugated monoclonal anti-BrdU antibody (substrate TMB). The optical density was recorded as $450 / 620 \mathrm{~nm}$ absorption ratio in an ELISA plate reader (Bio-Rad). DNA from sucrose fed mosquito's midguts cultivated with BrdU was used to blank readings. DNA from baker yeast grown in PBS solution mixed with $0.1 \mathrm{~g} \mathrm{LB}$ powder media and BrdU $100 \mu \mathrm{g} / \mathrm{ml}$ (for $48 \mathrm{~h}$ ) was used as a positive control. DNA from yeast grown without BrdU and mosquito's midguts BrdU-sucrose fed were used to normalization by assigning a value of 1 to the mean ratio.

Cisplatin (kind gift from Dr. Vicente Madrid, CISEI-INSP), a known anti-tumor agent, inhibits DNA duplication in silkworm larval midguts, without apoptotic effects (Hasinoff et al., 2004, 2005; Bragado et al., 2007; Huang et al., 2016). In order to inhibit DNA synthesis, 1 and 9-day post-infection, the Aedes aegypti females were fed overnight with cotton soaked with $100 \mu \mathrm{M}$ cisplatin/sucrose solution. Effect of the cisplatin treatment was assessed through three scrutiny points: visual confirmation of ovary shape and egg bunch formation, mortality ratio and counting the number of eggs laid.

\section{Bio-Informatics Strategy}

Drosophila melanogaster endoreplication mechanism involves the Delta-Notch signaling pathway, the main molecules involved in this process were obtained from $\mathrm{KEGG}^{1}$ and Flybase $^{2}$ databases (Supplementary Table 1).

We searched the transcript coding for Aedes aegypti hindsight molecule in VectorBase database ${ }^{3}$, Aedes aegypti Liverpool Strain, AaegL3 and AaegL3.4, genome and transcriptome respectively (Giraldo-Calderón et al., 2015). FBpp0070648 peptide and FBgn0003053 nucleotide sequences from Drosophila melanogaster database ${ }^{2}$ (Gramates et al., 2017) were used as bait, screening the afore mentioned databases using BLAST program (default parameters).

To localize orthologues, OrthoDB online program was used (Zdobnov et al., 2017), and for protein properties (family, domains and repeats, gene ontology term prediction and protein architecture) InterproScan website was used (Finn et al., 2017). In silico translation were performed with Exonerate generic tool. Exhaustive dynamic programming algorithm was employed to find the most likely hnt putative coding sequence in Aedes aegypti genome (Slater et al., 2005). Reverse translation allowed us to find the genome coordinates of hnt. The complete genomic region was screened for coding-sequences (CDS) using mRNA prediction tools like Augustus, FGenesh, GeneID, GeneMark, and GenScan (Burge and Karlin, 1997; Salamov and Solovyev, 2000; Besemer and Borodovsky, 2005; Blanco et al., 2007; Keller et al., 2011).

\section{Phylogenic Tree}

The sequences of hnt and mammal orthologue ras responding binding element-1 (Ming et al., 2013) were aligned using Multiple Sequences Alignments MUSCLE tool (Edgar, 2004).

The sequences were obtained from Vectorbase database $^{3}$ (Anopheles albimanus AALB003334, Anopheles gambiae AGAP000984, Aedes albopictus AALF021323, Culex quinquefasciatus CPIJ017756, Stomoxys calcitrans SCAU012431, Musca domestica MDOA011777, Glossina morsitans GMOY008594, Glossina brevipalpis GBRI040699); Flybase database ${ }^{2}$ (Drosophila melanogaster FBgn0003053), Ensembl database ${ }^{4}$ (Apis mellifera GB51515, Tribolium castaneum TC009560), NCBI database ${ }^{5}$ (Bombus terrestris nuccore/1185570083, Lucilia cuprina protein/906472602, Homo sapiens protein/51173735, Mus musculus protein/85719305.

Phylogenetic tree was constructed using PhyML software v3.0 running an algorithm of maximum-likelihood allowing 1,000 bootstrap sample repetitions (Guindon et al., 2010; Lefort et al., 2017), the protein tree was visualized and optimized using FigTree tool v1.4. $3^{6}$.

Sequences obtained and aligned were subjected to Blast2GO for Gene Ontology Terms and Interpro domains features.

\footnotetext{
${ }^{1}$ http://www.kegg.jp/dbget-bin/www_bget?aag04330

${ }^{2}$ www.flybase.org

${ }^{3}$ www.vectorbase.org

${ }^{4}$ www.ensembl.org

${ }^{5}$ www.ncbi.nlm.nih.gov

${ }^{6}$ http://tree.bio.ed.ac.uk/software/figtree/
} 


\section{Putative Protein Architecture and Domain Features Determination}

Deduced Hnt and RREB-1 aminoacidic sequences were analyzed with Interpro web tool (Finn et al., 2017), families and domains from the sequences were aligned to construct a visual signature of conserved features like zinc finger, zinc finger like and zinc finger-DNA binding domain, GBlocks tool was used to delete poorly aligned positions and divergent regions of the sequences (Castresana, 2000; Dereeper et al., 2008). A visual representation of the four main clusters with double zinc finger domain was made (Ming et al., 2013).

\section{Primer Design}

NCBI Primer design tool was used to search for candidate primers for amplification of part of the Aedes aegypti hnt putative coding regions (Ye et al., 2012), Oligo Analyzer online tool was checked for physicochemical properties (Owczarzy et al., 2008). Hnt PCR products were sequenced and aligned to confirm Aedes aegypti hnt putative coding region.

Ribosomal protein S7 (AAEL009496) 292 bps amplicon; forward 5' GGG ACA AAT CGG CCA GGC TAT C 3', reverse $5^{\prime}$ TCG TGG ACG CTT CTG CTT GTT G 3' primers were used for internal control PCR (Xi et al., 2008).

From CDS and mRNA predicted sequences, following primers were designed: $h n t . B$ (Fwd): 5' CGC AAG GAG TTA GAG CGT GA 3', hnt.B (Rev): $5^{\prime}$ GTG TCG ATC GCA GTT GGA CT $3^{\prime}$; hnt.C (Fwd): 5' AGT CCA ACT GCG ATC GAC AC 3', hnt.C (Rev): 5' CTT TCC ACC CCG ACA ACC TT 3'). Aedes aegypti L-4 larvae total RNA was used to perform hnt RT-PCR endpoint assay and PCR product nucleotide sequencing in Synthesis Unit and Sequencing of Institute of Biotechnology, UNAM. México.

The primers designed to amplify DENV universal region were DENV_all (Fwd) 5' - CAA TAT GCT GAA ACG CGA GAG AA3', DENV_all (Rev) 5'- CCC CAT CTA TTC AGA ATC CCT $\mathrm{GC}-3^{\prime}$ for a 171 bps amplicon. A third primer was used to amplify DENV serotype $25^{\prime}$ - TGC TGT TGG TGG GAT TGT TA $-3^{\prime}$ for a 150 bps amplicon. The quantitative PCR of DENV2 infection were normalized using a quantified plasmid with an insert of DENV-2 genome as template (kindly donated by Dr. Rosa Ma. Del Angel, CINVESTAV, IPN).

\section{Statistical Analysis}

Infection kinetics assays were performed six times. Data from all quantitative assays were subjected to D' Agostino-Pearson and Shapiro-Wilk normality tests, if positive parametric comparisons to test differences between infected/non-infected samples, One-way ANOVA and, when required, Geisser-Greenhouse's correction was used (to eliminate possible data sphericity). Data unpaired assessments Student's T with Welch's correction for infection kinetics was performed.

Non-parametric assays were Kruskal-Wallis and MannWhitney tests, data were verified with Kolmogorov-Smirnov test, for robust analysis. All data were subjected to a $P$-value $<0.05$ for a significative difference. Data were analyzed, and graphics made in GraphPad Prism v6.01.

\section{RESULTS}

\section{Dengue Virus Feeding Induce DNA Synthesis in Anopheles albimanus and Aedes aegypti}

DNA synthesis de novo has been observed in Anopheles albimanus exposed to diverse bacterial, yeast and protozoarious immune challenges (Hernandez-Martinez et al., 2006; Hernández-Martínez et al., 2013; Contreras-Garduño et al., 2015). We challenged the two mosquitoes with DENV, using the BrdU incorporation as de novo DNA synthesis marker. We identified midgut cells undergoing DNA synthesis phase without entering mitosis phase. The midgut and abdomen cells respond to the viral challenge initiating DNA synthesis.

We tracked BrdU fluorescence upon viral challenge in Anopheles albimanus and Aedes aegypti mosquitoes during seven days. Since Anopheles albimanus (a non-Dengue virus vector) DNA synthesis during pathogen insult has been previously described, we used this insect as a comparative model organism for BrdU incorporation. When challenged with Dengue virus, both Anopheles albimanus and Aedes aegypti mosquitoes were incorporating BrdU into their midguts and abdomens nuclei. We observed BrdU nuclear signal in Anopheles albimanus and Aedes aegypti, 5 and 7 days post virus alimentation respectively (Figures 1A,B).

Aedes aegypti BrdU positive midgut cells proportion in the distinct treatments were the following: at 3 and 5 days post infection, BrdU positive cells number were virtually identical in Mock and DENV fed Aedes aegypti intestines. Seven days post alimentation, DENV fed mosquitoes intestines showed a steep increase in BrdU intestine positive cells (35\% of the cell were positive) while blood fed control mosquitoes showed no such increase ( $1 \%$ of labeled cells). The proportion of BrdU positive cells in the whole abdomen followed the same tendency [DENV/Mock 3 dpi (0/0), 5 dpi (7/5), 7 dpi (41/1)], showing an even larger proportion of positive cells seven days post infection (Figure 1C).

Anopheles albimanus is a mosquito capable to endoreplicate genomic DNA to fight fungi, bacterial and parasite infections (Hernandez-Martinez et al., 2006; Hernández-Martínez et al., 2013; Contreras-Garduño et al., 2015). DENV does not infect naturally Anopheles albimanus (Ramos-Castañeda et al., 2008). Anopheline mosquitoes natural refractoriness mechanisms to DENV are unknown. Nevertheless, it is noteworthy that DENV challenge induced a faster DNA synthesis in Anopheles albimanus (3 days post-challenge) than in Aedes aegypti (7 days postchallenge).

\section{Aedes aegypti infections With Dengue Virus Triggers DNA synthesis}

Aedes aegypti were fed with BrdU and 3 days later infected with DENV-2. A kinetics experiment was performed dissecting the mosquitoes 3, 5, 7, 10, and 14 days post infection/alimentation (control). The midguts of the infected mosquitoes showed a peak DNA synthesis 7 days post infection, corresponding to the point of highest viral 
A

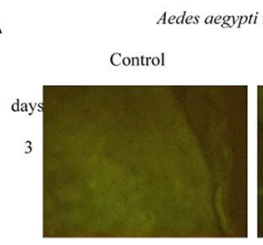

edes aegypti midguts

Anopheles albimanus midguts
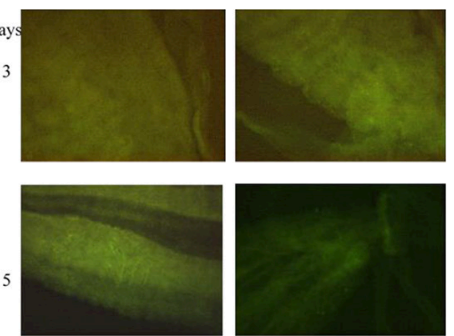

Control

DENV-2
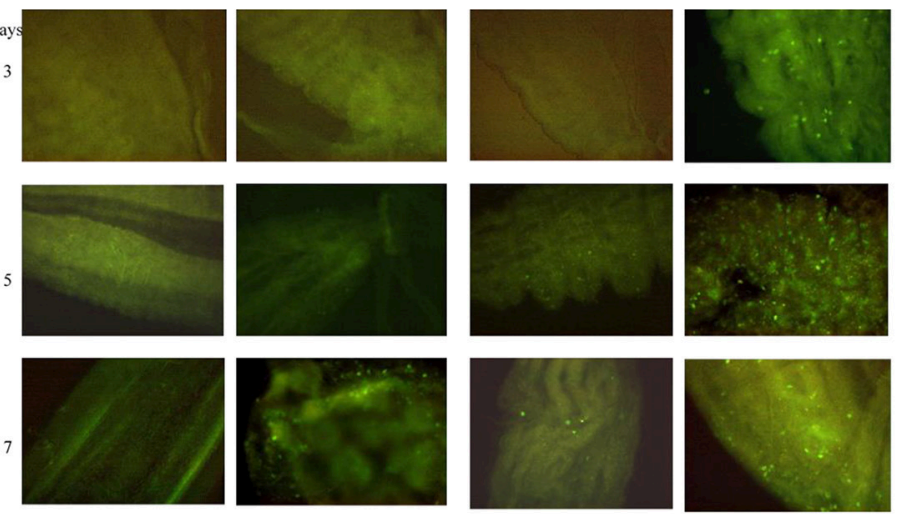

B

Aedes aegypti abdomens

Anopheles albimanus abdomens

Control

DENV-2

Control

DENV-2
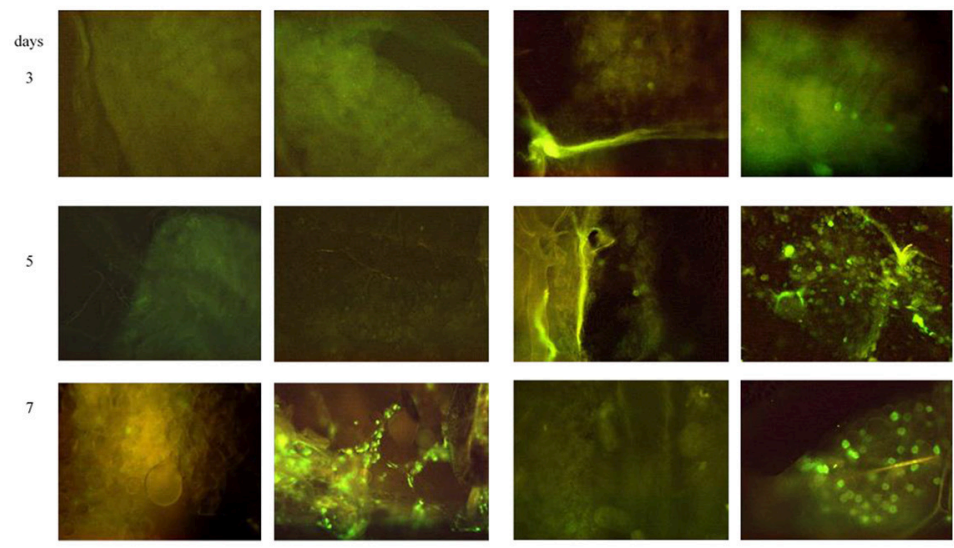

C

Aedes aegypti

Anopheles albimanus
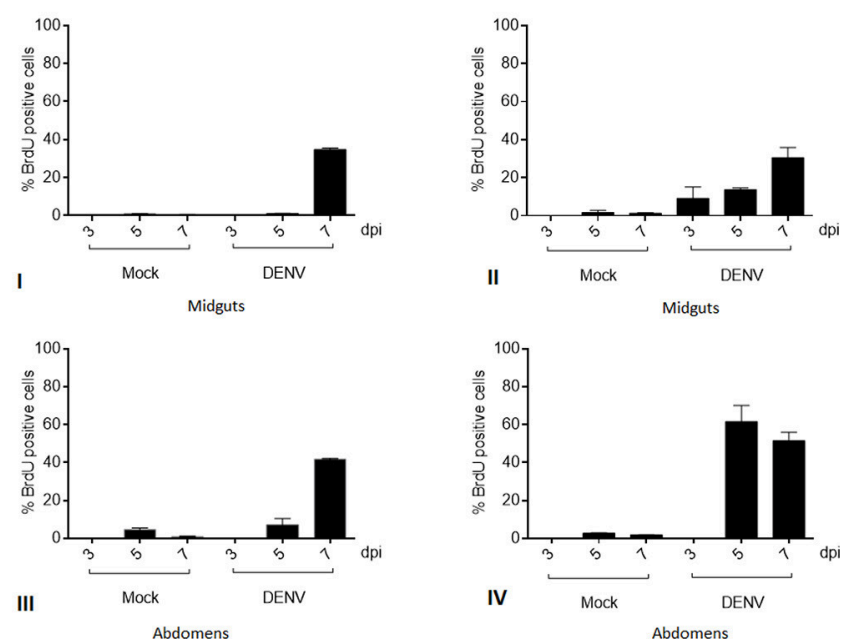

FIGURE 1 | (A) DNA synthesis in mosquitoes challenged with Dengue virus by in situ immunofluorescence assay. Viral challenged tissues respond by endoreplicating genomic DNA. Control challenged tissues show no evidence of DNA synthesis. Anopheles albimanus was used as a positive control for DNA synthesis. Intense DNA synthesis is observed in Anopheles albimanus at earlier times, meanwhile Aedes aegypti response delays until 7 days. Aedes aegypti midguts control (erythrocytes plus MEM media) and DENV challenged. Anopheles albimanus midguts control and DENV challenged. (B) DNA synthesis in mosquitoes challenged with Dengue virus by in situ immunofluorescence assay. Aedes aegypti abdomens control (erythrocytes plus MEM media) and DENV challenged. 
FIGURE 1 | Anopheles albimanus abdomens control and DENV challenged. (C) DNA synthesis in mosquitoes challenged with Dengue virus by in situ immunofluorescence assay. BrdU positive cell percentage per microscope field: of Aedes aegypti midgut cells (I) or abdomen cells (III) 3 , 5 , and 7 days post blood (mock) or blood plus $1 \times 10^{8} \mathrm{FFU} / \mathrm{mL}$ DENV alimentation. BrdU positive cell percentage per microscope field: of Anopheles albimanus midgut cells (II) or abdomen cells (IV) 3, 5, and 7 days post blood (mock) or blood plus $1 \times 10^{8} \mathrm{FFU} / \mathrm{mL}$ DENV alimentation.

load, as shown by FFU assay and viral genome copies (Figures 2A-C). Nevertheless, by day 10 post alimentation; 3 days after the DNA synthesis peak; the viral load diminishes.

\section{In Silico Search for Delta-Notch Signaling Orthologs in Aedes aegypti}

In Drosophila melanogaster, endoreplication mechanism involves the Delta-Notch signaling pathway. In the fruit fly, the DNA replication has been amply described during oogenesis. More recently this phenomenon has been observed in the midgut during stress challenges. The Notch signal transduction cascade is responsible for the endocycles observed in the Drosophila midgut cells (Lee et al., 2010; Edgar et al., 2014).

Therefore, we searched for Aedes aegypti orthologs of the known D. melanogaster proteins involved in the Notch canonical pathway. Aside from Hindsight (hnt) gene, all the Notch canonical pathway main proteins were identified in Aedes aegypti transcriptome (Supplementary Table 1).

Hindsight is responsible for the transition from mitotic cycle to endocycle, degrading cytoplasmic cyclins and also functioning as a transcriptional factor (Yip et al., 1997; Baechler et al., 2016). Using the peptidic sequence of Drosophila hnt as bait, we performed an in-silico translation corresponding to Aedes aegypti hnt. Positive matches were found in the supercontigs 1.787 and 1.430 regions, formerly described as non-coding.

We used exhaustive dynamic programming of the Exonerate generic tool to obtain an Aedes aegypti hnt putative protein. Reverse translation of the putative $h n t$ protein allowed us to map the gene coordinates in the supercontigs 1.787 and 1.430 (Supplementary Figure 1).

The sequences of invertebrate hnt and vertebrate orthologue RREB-1 were aligned using MUSCLE tool (Edgar, 2004). A phylogenetic tree was constructed (Figure 3A). The Culicid family grouped in one clade, other insects (bee, bumblebee, beetle) grouped in another clade (arthropods), and flies grouped in third clade (Guindon et al., 2010; Lefort et al., 2017). This tree shows the domains of this protein that are genuinely conserved between those species. Blast2GO output file shows that all hnt/ $R R E B-1$ proteins share the same putative function. Results data were consistent in GO terms (Supplementary Data Sheet 1). The $H n t / R R E B-1$ protein family requires the presence of four zincfinger cluster doublets in order to bind to its DNA elements (Ming et al., 2013). A map detailing the protein architecture is shown in Supplementary Figure 2.

An exon prediction analysis was made using five different tools: Augustus (Keller et al., 2011), FGenesh (Salamov and Solovyev, 2000), GeneID (Blanco et al., 2007), GeneMark (Besemer and Borodovsky, 2005) and GenScan (Burge and Karlin, 1997). Hnt CDS and mRNA coding regions were deduced and oligonucleotide primers for $h n t$ amplification were designed, sequences in Supplementary Tables 2, 3 (Zanchi et al., 2011).

\section{Aedes aegypti Hnt, Delta, and Notch Transcription Levels During DENV Infection}

The Hnt PCR results of the mosquito genomic DNA and the cDNAs of the different experimental conditions are congruent with the in silico analysis results: the sizes of the respective PCR (genomic and cDNA) are consistent with the processing of the predicted intron (Figure 3B). Considering that we detected a Hnt transcript suitable for translation, this result further sustains the existence of the putative Hnt protein.

We analyzed Hnt, Delta and Notch transcription through quantitative RT-PCR experiments (Figures 3C-E). 3 days postinfection we observed that Delta and Notch transcriptions increase and remain higher than in mock-infected samples. This might be reflecting the onset of a strategy to prepare the DNA replication machinery for later activation. Hnt gene showed a peak transcription 7 days post-infection and remains overtranscribed until the 10th day. On the 14th day, Hnt transcription decreases to the levels of mock-infected mosquitoes (Figure 3D). The phasing of those events: first the viral challenge, then the transcription increased of those three genes, and finally the de novo DNA synthesis support the assumption of a causal link between those events. Also, the DNA synthesis is concomitant to virus load abatement, suggesting that either the DNA replication or the hnt protein synthesis are favoring antiviral activity.

\section{DNA Synthesis Inhibition Affect the Susceptibility of the Aedes aegypti Mosquito to Dengue}

Cisplatin is a known anti-cancer agent, preferentially binding to guanine base. It interferes with DNA replication forming crosslinked DNA adducts. At high cisplatin doses $(100 \mathrm{mM})$, inhibition of DNA replication leads to apoptosis of human cancerous cell line (Hasinoff et al., 2004). At low doses $(100 \mu \mathrm{M})$, it also inhibits DNA duplication in Bombyx mori (Huang et al., 2016). We assessed the impact of cisplatin oral-feeding, 1 and 9-days post blood-feeding, on Aedes aegypti fitness. There were no differences in mosquito mortality between the $100 \mu \mathrm{M}$ cisplatin treated and non-treated groups (data not shown). The sole side-effect observed was a diminished egglaying between treated groups. Cisplatin treated mosquitoes had a lowered egg production, when compared to untreated groups (Supplementary Figure 3). Visual inspection showed that cisplatin fed mosquitoes ovarian tissues were similar to the control ovarian tissue (data not shown).

We determined the impact of cisplatin treatment on Dengue virus infection susceptibility of Aedes aegypti. Since cisplatin is known to form DNA adducts at a $100 \mu \mathrm{M}$ concentration 


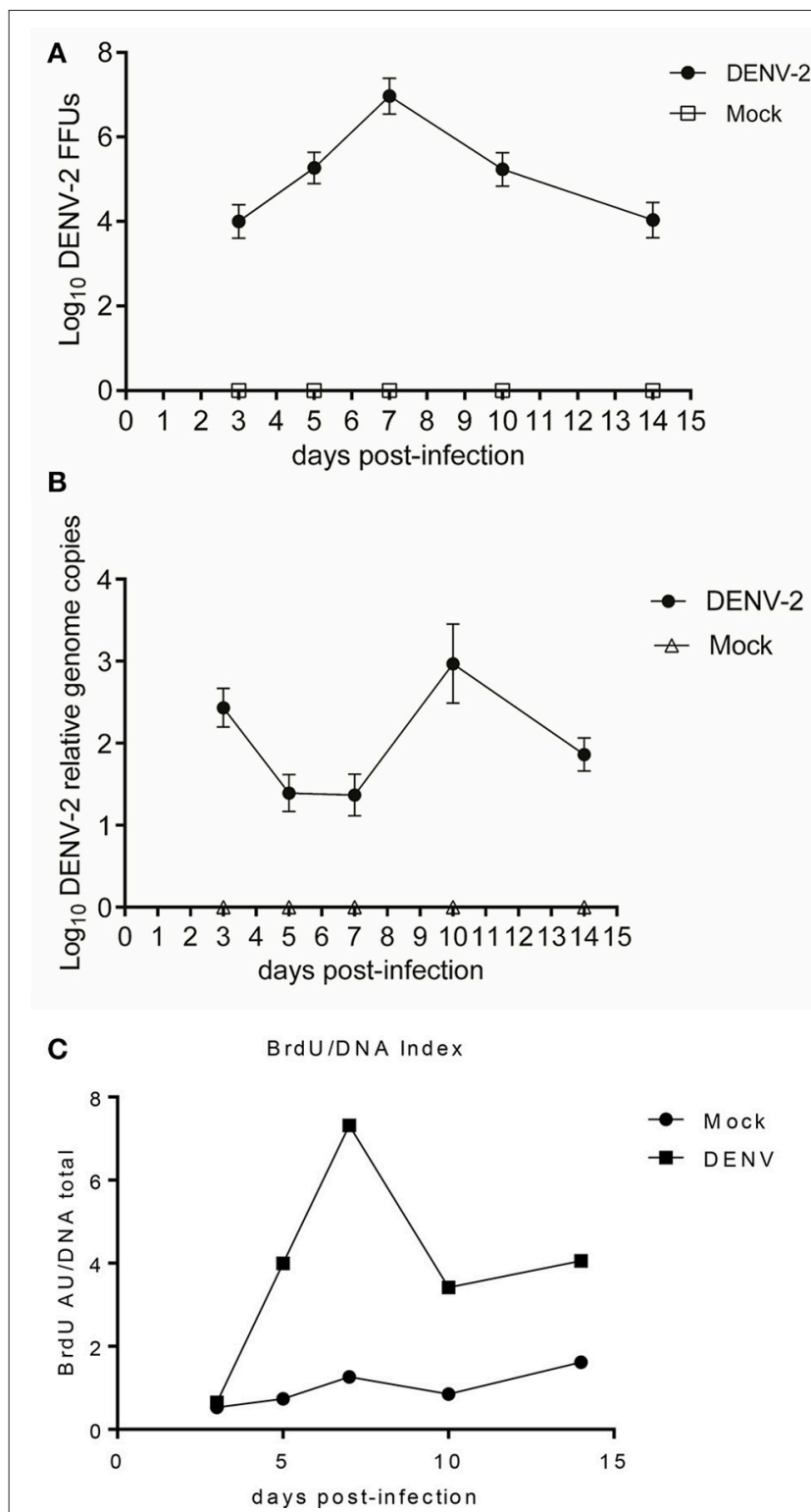

FIGURE 2 | (A) DENV-2 and mock-infected midguts kinetics at 3, 5, 7, 10, and 14 days post-infectious blood meal. Focus forming units assay. One-way ANOVA $F=886.0, p<0.0001$. DENV-2 vs. Mock Student's $T$-test with two-tailed Welch's correction: Midguts FFU of one mosquito 3, 5, 7, 10, and 14 dpi. All comparisons had statistically significant difference $t=30.47$, 43.0, 49.25, 39.56, 29.13; $p<0.0001$. (B) DENV-2 and mock-infected midguts kinetics at $3,5,7,10$, and 14 days post-infectious blood meal. Viral genome copies from DENV-2 and mock-infected midguts lysate. One-way ANOVA $F=299.5, p<0.0001$. DENV-2 vs. Mock Student's $T$-test with Welch's correction two-tailed: $3,5,7,10$, and 14 dpi all comparisons with significant difference $t=30.91,18.49,16.27,18.49$, and $27.71 ; p<0.0001$. (C) DENV-2 and mock-infected midguts kinetics at $3,5,7,10$, and 14 days post-infectious blood meal. Kinetic of the Relative BrdU absorbtion/DNA quantity index. We determined the ratio between BrdU signal and total DNA amount, for the DENV-2 and mock-infected midguts over 14 days post infectious/mock blood meal. ELISA for BrdU incorporation. Kruskal-Wallis test $=72.83, p<0.0001$, DENV-2 vs. Mock Mann Whitney Test two-tailed: 3 dpi no statistically significant differences $=0.22, p=0.1144 ; 5,7,10$, and 14 dpi comparisons with statistically significant differences $=6.83,10.16,6.36,5.19 ; p=0.0002$.
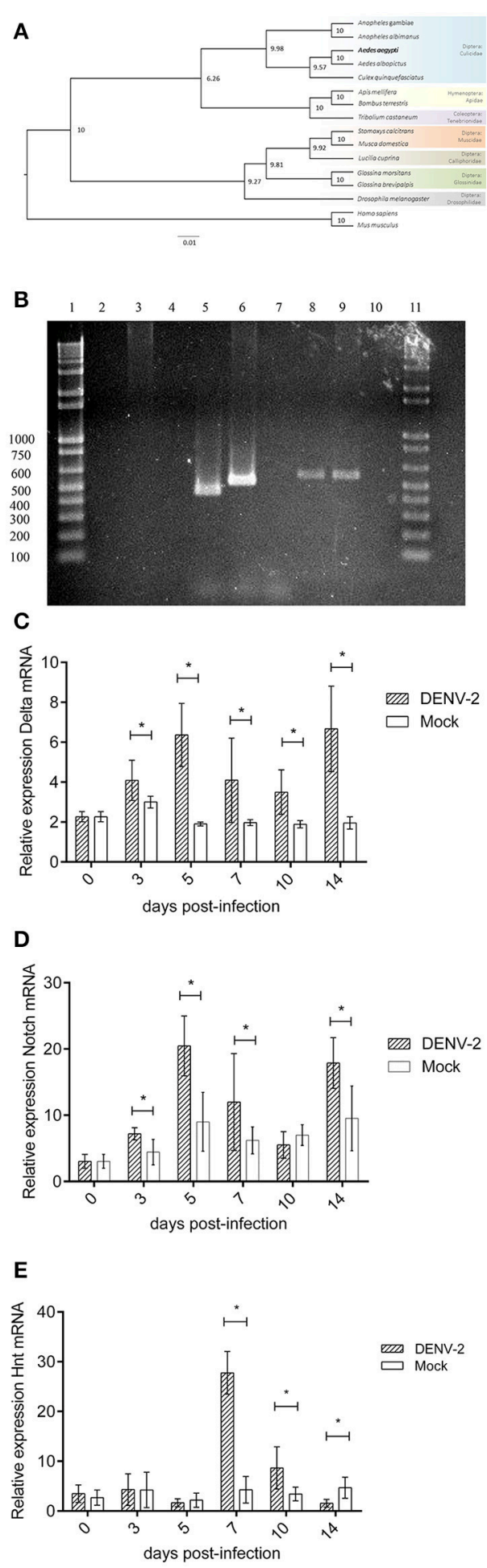

FIGURE 3 | (A) Notch pathway transcriptional analysis during DENV infection in Aedes aegypti midguts. Hindsight and RREB-1 phylogenetic tree based on maximum-likelihood algorithm. The sequences shown were aligned using MUSCLE tool. The phylogenetic tree was constructed using PhyML software v3.0 with 1,000 bootstrap sample repetitions. (B) Notch pathway

(Continued) 
FIGURE 3 | transcriptional analysis during DENV infection in Aedes aegypti midguts. Hindsight PCR from mosquito midguts gDNA and cDNA. Lanes, (1) $3 \mathrm{~Kb}$ Ladder. (2) cDNA (mRNA)-Hnt A amplicon. (3). gDNA-Hnt A amplicon. (4) No template control-Hnt A amplicon. (5) cDNA (mRNA)-Hnt B amplicon. (6) gDNA-Hnt B amplicon. (7) No template control-Hnt B amplicon. (8) cDNA (mRNA)-Hnt C amplicon. (9) gDNA-Hnt C amplicon. (10) No template control-Hnt C amplicon. (11) $3 \mathrm{~Kb}$ Ladder. (C) Notch pathway transcriptional analysis during DENV infection in Aedes aegypti midguts. Relative expression of Delta transcript 0, 3, 5, 7, 10, and 14 days post alimentation with blood (Mock) or blood with $1 \times 10^{8}$ DENV2 FFU/mL (DENV-2). Mock vs. DENV-2 Student's $T$-test with Welch's correction 0, 3, 5, 7, 10, 14 dpi $t=0.0$, 3.58, $9.74,3.47,4.93$, and 7.55, $p>0.9999,0.0055,<0.0001,0.0082,0.0010$, $<0.0001$. (D) Notch pathway transcriptional analysis during DENV infection in Aedes aegypti midguts. Relative expression of Notch transcript 0, 3, 5, 7, 10, and 14 days post alimentation with blood (Mock) or blood with $1 \times 10^{8}$ DENV2 FFU/mL (DENV-2). Mock vs. DENV-2 Student's $T$-test with Welch's correction $0,3,5,7,10,14$ dpi $t=0.0,4.51,6.25,2.63,2.04,4.66, p>$ $0.9999,0.0008,<0.0001,0.0267,0.0592,0.0003$. (E) Notch pathway transcriptional analysis during DENV infection in Aedes aegypti midguts. Relative expression of Hindsight transcript 0, 3, 5, 7, 10, and 14 days post alimentation with blood (Mock) or blood with $1 \times 10^{8}$ DENV2 FFU/mL

(DENV-2). Mock vs. DENV-2 Student's T-test with Welch's correction 0, 3, 5, 7,10 , and 14 dpi $t=1.18,0.29,1.03,16.04,4.06,4.77, p=0.2539,0.7749$, $0.3191,<0.0001,0.0024,0.0007$.

(thereby impeaching the DNA strands to be separated), the adduct formation would hinder DNA endoreplication. The cisplatin treated mosquitoes did show a higher viral load as well as a higher infective virus presence. Upon infection (Figures 4B,C), their DNA BrdU incorporation was diminished when compared to non-treated infected mosquitoes (Figure 4A). This experiment provides a positive link between the mosquito genomic DNA endoreplication phenomenon and the resistance to viral infection.

Also, in cisplatin treated mosquito group, hnt gene relative transcription was reduced to levels comparable to control groups (Figure 4D). Inhibition of $h n t$ transcription would hinder the de novo DNA synthesis, hence inhibiting the potential antiviral mechanism hereby proposed; leaving viral replication unrestricted.

\section{DISCUSSION}

The genomic DNA endoreplication phenomenon has been observed in insect during development and stress response. In these conditions, endoreplication of genes related to macromolecules synthesis pathway enhance their production, hence favoring the organism growth or the cell stress resistance. This work explores the genomic response of Aedes aegypti to the DENV infection. Transcriptomic and microarray analysis between Aedes aegypti strains with high and low DENV susceptibility show a common core of active genes, indicating a functional signal transduction cascade activating the host response. Nevertheless, upon virus infection, the low-susceptibility strain showed up-regulation genes involved in several active metabolic processes (glycolysis, gluconeogenesis, cell cycle progression and differentiation). The viral resistance may be related to cell cycle alteration (Behura et al., 2011; Chauhan et al., 2012).

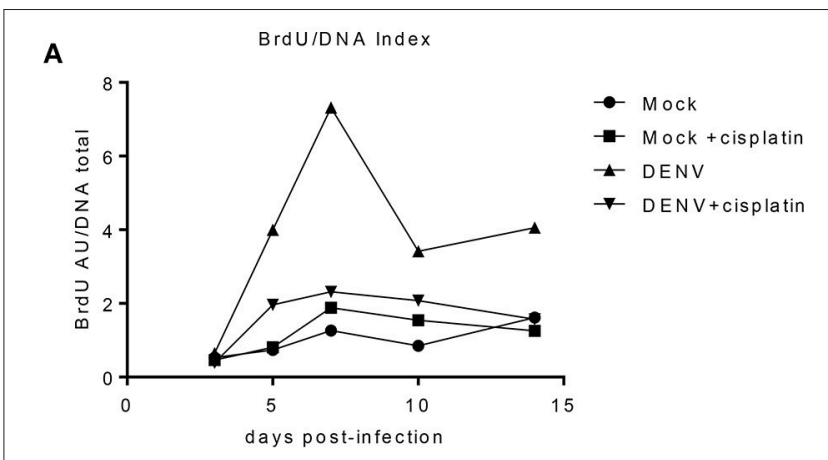

B
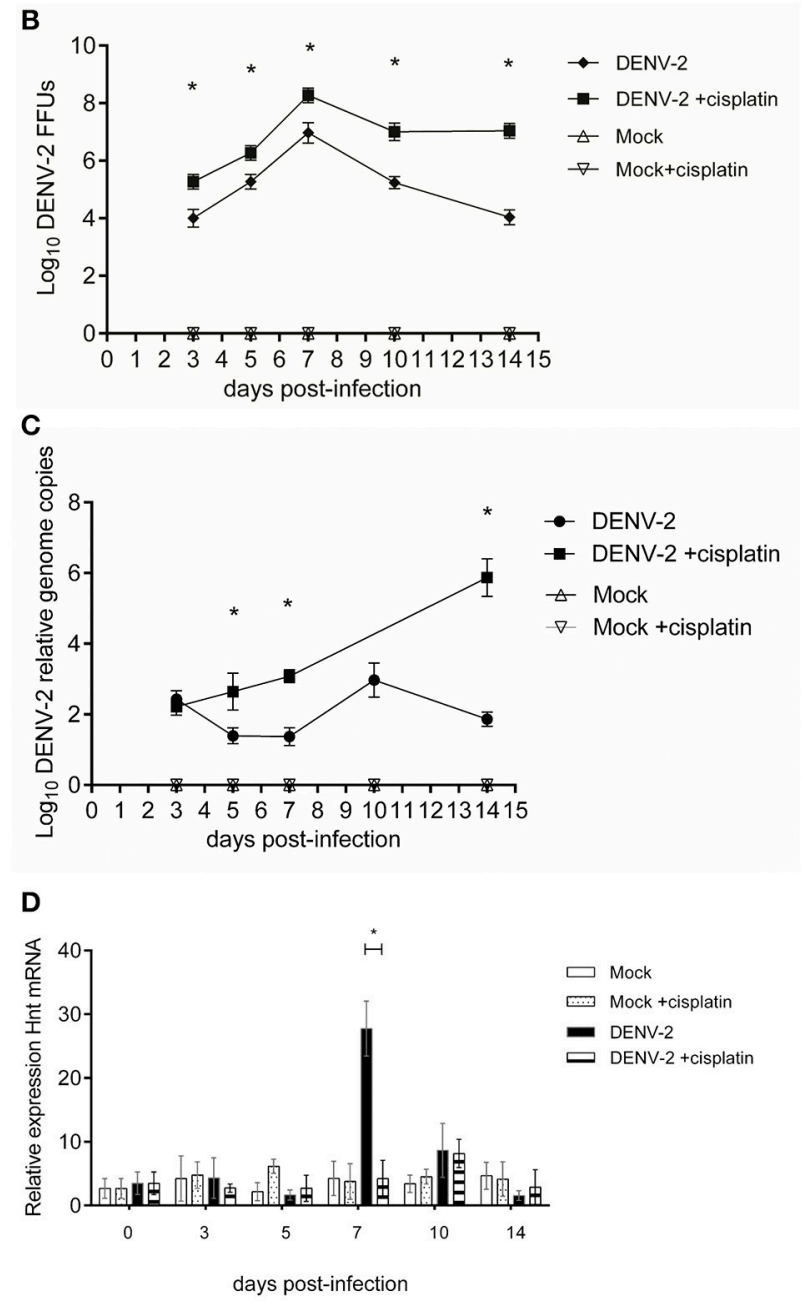

FIGURE 4 | Cisplatin treated and untreated DENV-2/mock infected midguts kinetics at 3, 5, 7, 10, and 14 days post-infectious blood meal. (A) normalized BrdU incorporation, in Cisplatin treated and untreated DENV-2/mock infected midguts. The midguts were extracted at $3,5,7,10$, and 14 days post-infectious blood meal. ELISA for BrdU incorporation. Kruskal-Wallis test $=129.6, p<0.0001$, Mock vs. Mock +cisplatin Mann Whitney two-tailed test: non-significative difference $=0.025,-0.035,0.265,0.735,0.575$, $p=0.7576,0.0085,>0.9999,0.7026,0.1563,0.3162 ;$ DENV vs. DENV +cisplatin Mann Whitney two-tailed test: all comparisons significative difference $3,5,7,10,14$ dpi: $-0.56,-4.70,-7.72,-4.06,-5.15$; $p=0.0085,0.0421,0.0002,0.0118,0.0022$. DENV vs. DENV + cisplatin Kolmogorov-Smirnov test: 3 and 5 dpi no-significative difference $=0.6250$,

(Continued) 
FIGURE $4 \mid 0.5, p=0.0536,0.2827 .7,10$, and 14 dpi showed significative difference $=1,0.75,0.75, p=0.0002,0.0186$, and 0.0186 . Two tests were realized for robust analysis. (B) Focus forming units assay of Cisplatin treated and untreated DENV-2/Mock infected midguts extracts; $3,5,7,10$, and 14 days post-infectious blood meal. One-way ANOVA $F=104.4, p<0.0001$. DENV-2 vs. DENV-2 cisplatin treatment, 3, 5, 7, 10, and 14 dpi Student's $T$-test with two-tailed Welch's correction: all comparison with significative difference $t=6.42,5.22,6.38,9.8,15.32 ; p<0.0001$. (C) Viral genome copies determination of Cisplatin treated and untreated DENV-2/Mock infected midguts extracts; $3,5,7,10$, and 14 days post-infectious blood meal. Viral genome copies. One-way ANOVA with Geisser-Greenhouse's correction epsilon $=0.1651, F=170.2, p<0.0001$. DENV-2 vs. DENV-2+cisplatin treatment Student's $T$-test with two-tailed Welch's correction 3 dpi $t=1.95$, $p=0.0679$ no significative difference: 5,7 , and 14 dpi comparisons with significative difference $t=6.63,16.42,21.09 ; p<0.0001$. (D) Relative expression of $\mathrm{Hnt}$ transcript in the mosquito cells from Cisplatin treated and untreated DENV-2/Mock infected midguts. The kinetics were performed at 3 , $5,7,10$, and 14 days post-infectious blood meal. Mock vs. Mock +cisplatin Student's $T$-test with Welch's correction 3, 5, and 10 dpi significative difference. DENV-2 vs. DENV-2 +cisplatin Student's T-test with Welch's correction only 7 dpi showed significative difference $p<0.0001$.

During viral challenge, we observed a nucleotide incorporation in the mosquitoes midguts. The inhibition of DNA replication through cisplatin treatment led to a higher virus load in the mosquitoes, thereby linking the antiviral response to the endoreplication process.

The mechanism underlying the phenomenon was explored tracking the transcription of putative Aedes aegypti ortholog genes of Drosophila Delta-Notch signaling pathway. We also quantified $h n t$ transcription during the endoreplication process. We found a positive correlation between BrdU incorporation, the viral titer peak and $h n t$ transcript increase.

Through unknown pathways, DENV infection actively repress the antimicrobial peptide production of Aedes aegypti, During the infection, the Jak/STAT, Toll, IMD and RNA interference pathways are activated in Aedes aegypti, supposedly through danger sensing signal cascade. This activation would provide the ability to limit viral replication and spreading in the mosquito.

There are few published works that explore global transcriptomic response between Aedes aegypti strains with differential vectorial competence. Hence, the mechanisms underlying these differences and ability to limit viral replication are unclear. The role of transcription factors involved in cell cycle progression, like Wnt, Hedgehog and Notch in virus inhibition has not been elucidated so far. Transcriptomics approaches only describes Notch transcript differences during infection; without linking viral spreading and cell cycle components (Behura et al., 2011; Chauhan et al., 2012). The Notch pathway has been implicated in host immune response against DENV in human (Li et al., 2015), so we speculated that the transcription of Notch cascade components could also be triggered in the mosquito, leading to a posterior DNA synthesis. Indeed, in Aedes aegypti, Delta ligand was found to be differentially over expressed during DENV infection (Colpitts et al., 2011).
Upon DENV infection, we observed increased transcription of hnt gene. The hnt gene expression is regulated by the DeltaNotch signaling pathway. This pathway is also transcriptionally affected by viral infection. We found that Aedes aegypti Delta, Notch and hnt transcripts were overexpressed during DENV infection. Post infection, we observed BrdU nucleotide base analog incorporation in the intestinal cells of the mosquito, implying that an active DNA replication is taking place in these cells. Since there is no cell proliferation in adult mosquitoes, we supposed that this phenomenon could be due to endoreplication process. Hnt protein has been described to be responsible for Drosophila endoreplication processes (Sun and Deng, 2007), and recent work described active transcription of the $h n t$ putative gene in Aedes albopictus infected with DENV-2 (Tsujimoto et al., 2017). We found the Aedes aegypti homolog of Aedes albopictus hindsight and observed an over-transcription of this gene during infection. Inhibition of endoreplication process through DNA adduct formation enhanced viral infection, thereby underscoring the relevance of this cellular process in containing the viral infection.

Our results indicate that Notch pathway is activated during DENV infection in Aedes aegypti and endoreplication seems to be part of the mosquito response against DENV. Understanding the biological activity of Notch and its role in endoreplication of disease-transmitting mosquitoes will help to generate new strategies for controlling disease transmission. The molecular mechanisms that induce and regulate the activation of Notch pathway require further investigation.

\section{AUTHOR CONTRIBUTIONS}

JS-S, SH-M, JM-B, RC, AA-D, FZ-E and HL-M conceived and design experiments. JS-S, SH-M, and AA-D performed the experiments. JS-S, FZ-E, and JM-B bio-informatics analysis. SH$\mathrm{M}$ and HL-M contributed reagents, materials, analysis tools. JS-S, $\mathrm{RC}$, and HL-M wrote the paper.

\section{ACKNOWLEDGMENTS}

This work was supported by the National Council of Research (Conacyt) with the grant number 243171.

JS-S received Doctorado en Ciencias en Enfermedades Infecciosas (CONACYT PhD Grant No. 299687). We thank Dr. Mario Henry Rodriguez-López and Dr. Ma Isabel SalazarSánchez for their helpful comments that helped improving this paper, FZ-E for phylogenetic tree execution and Krystal Maya Maldonado for cisplatin experiment evaluation.

\section{SUPPLEMENTARY MATERIAL}

The Supplementary Material for this article can be found online at: https://www.frontiersin.org/articles/10.3389/fmicb. 2018.00801/full\#supplementary-material 


\section{REFERENCES}

Baechler, B., McKnight, C., Pruchnicki, P. C., Biro, N. A., and Reed, B. H. (2016). Hindsight/RREB-1 functions in both the specification and differentiation of stem cells in the adult midgut of Drosophila. Biol. Open 5, 1-10. doi: 10.1242/bio.015636

Baer, A., and Kehn-Hall, K. (2014). Viral concentration determination through plaque assays: using traditional and novel overlay systems. J. Vis. Exp. e52065. doi: 10.3791/52065

Behura, S. K., Gomez-Machorro, C., Harker, B. W., deBruyn, B., Lovin, D. D., Hemme, R. R., et al. (2011). Global cross-talk of genes of the mosquito Aedes aegypti in response to dengue virus infection. PLoS Negl. Trop. Dis. 5:1385. doi: 10.1371/journal.pntd.0001385

Besemer, J., and Borodovsky, M. (2005). GeneMark: web software for gene finding in prokaryotes, eukaryotes and viruses. Nucleic Acids Res. 33, W451-W454. doi: $10.1093 /$ nar/gki487

Blanco, E., Parra, G., and Guigó, R. (2007). Using GeneID to Identify Genes. Curr. Protoc. Bioinforma. Chapter 4, Unit 4.3. doi: 10.1002/0471250953.bi0403s18

Bragado, P., Armesilla, A., Silva, A., and Porras, A. (2007). Apoptosis by cisplatin requires p53 mediated p38alpha MAPK activation through ROS generation. Apoptosis 12, 1733-1742. doi: 10.1007/s10495-007-0082-8

Bubner, B., Gase, K., and Baldwin, I. T. (2004). Two-fold differences are the detection limit for determining transgene copy numbers in plants by real-time PCR. BMC Biotechnol. 4:14. doi: 10.1186/1472-6750-4-14 1472-6750-4-14 [pii]

Buchon, N., Broderick, N. A., Poidevin, M., Pradervand, S., and Lemaitre, B. (2009). Drosophila intestinal response to bacterial infection: activation of host defense and stem cell proliferation. Cell Host Microbe 5, 200-211. doi: 10.1016/j.chom.2009.01.003

Burge, C., and Karlin, S. (1997). Prediction of complete gene structures in human genomic DNA. J. Mol. Biol. 268, 78-94. doi: 10.1006/jmbi.1997.0951

Castagnola, A., and Jurat-Fuentes, J. L. (2016). Intestinal regeneration as an insect resistance mechanism to entomopathogenic bacteria. Curr. Opin. Insect Sci. 15, 104-110. doi: 10.1016/j.cois.2016.04.008

Castresana, J. (2000). Selection of conserved blocks from multiple alignments for their use in phylogenetic analysis. Mol. Biol. Evol. 17, 540-552. doi: 10.1093/oxfordjournals.molbev.a026334

Chan, A., Rodriguez, M. H., Torres, J., and Al., E. (1994). Susceptibility of Three Laboratory Strains of Anopheles albimanus (Diptera: Culicidae) to Coindigenous Plasmodium vivax in Southern Mexico. Entomol. Soc. Am. 37, 331-334. doi: 10.1093/jmedent/31.3.400

Chauhan, C., Behura, S. K., Debruyn, B., Lovin, D. D., Harker, B. W., GomezMachorro, C., et al. (2012). Comparative expression profiles of midgut genes in dengue virus refractory and susceptible Aedes aegypti across critical period for virus infection. PLoS ONE 7:e47350. doi: 10.1371/journal.pone.0047350

Cheng, G., Liu, Y., Wang, P., and Xiao, X. (2016). Mosquito Defense Strategies against Viral Infection. Trends Parasitol. 32, 177-186. doi: 10.1016/j.pt.2015.09.009

Colpitts, T. M., Cox, J., Vanlandingham, D. L., Feitosa, F. M., Cheng, G., Kurscheid, S., et al. (2011). Alterations in the Aedes aegypti Transcriptome during Infection with West Nile, Dengue and Yellow Fever Viruses. PLoS Pathog. 7:e1002189. doi: 10.1371/journal.ppat.1002189

Contreras-Garduño, J., Rodríguez, M. C., Hernández-Martínez, S., MartínezBarnetche, J., Alvarado-Delgado, A., Izquierdo, J., et al. (2015). Plasmodium berghei induced priming in Anopheles albimanus independently of bacterial co-infection. Dev. Comp. Immunol. 52, 172-181. doi: 10.1016/j.dci.2015.05.004

Dereeper, A., Guignon, V., Blanc, G., Audic, S., Buffet, S., Chevenet, F., et al. (2008). Phylogeny.fr: robust phylogenetic analysis for the non-specialist. Nucleic Acids Res. 36, 465-469. doi: 10.1093/nar/gkn180

Edgar, B. A., and Orr-Weaver, T. L. (2001). Endoreplication cell cycles. Cell 105, 297-306. doi: 10.1016/S0092-8674(01)00334-8

Edgar, B., a, Zielke, N., and Gutierrez, C. (2014). Endocycles: a recurrent evolutionary innovation for post-mitotic cell growth. Nat. Rev. Mol. Cell Biol. 15, 197-210. doi: 10.1038/nrm3756

Edgar, R. C. (2004). MUSCLE: multiple sequence alignment with high accuracy and high throughput. Nucleic Acids Res. 32, 1792-1797. doi: $10.1093 /$ nar/gkh340
Finn, R. D., Attwood, T. K., Babbitt, P. C., Bateman, A., Bork, P., Bridge, A. J., et al. (2017). InterPro in 2017-beyond protein family and domain annotations. Nucleic Acids Res. 45, D190-D199. doi: 10.1093/nar/gkw1107

Fox, D. T., and Duronio, R. J. (2013). Endoreplication and polyploidy: insights into development and disease. Development 140, 3-12. doi: 10.1242/dev.080531

Giraldo-Calderón, G. I., Emrich, S. J., MacCallum, R. M., Maslen, G., Dialynas, E., Topalis, P., et al. (2015). VectorBase: an updated bioinformatics resource for invertebrate vectors and other organisms related with human diseases. Nucleic Acids Res. 43, D707-D713. doi: 10.1093/nar/gku1117

Gramates, L. S., Marygold, S. J., Dos Santos, G., Urbano, J. M., Antonazzo, G., Matthews, B. B., et al. (2017). FlyBase at 25: looking to the future. Nucleic Acids Res. 45, D663-D671. doi: 10.1093/nar/gkw1016

Guindon, S., Dufayard, J.-F., Lefort, V., Anisimova, M., Hordijk, W., and Gascuel, O. (2010). New algorithms and mehtods to estimate maximum-likelihood phylogenies: asessing the performance of PhyML 2.0. Syst. Biol. 59, 307-321. doi: 10.1093/sysbio/syq010

Guo, Z., and Ohlstein, B. (2015). Bidirectional Notch signaling regulates Drosophila intestinal stem cell multipotency. Science 350:aab0988. doi: 10.1126/science.aab0988

Hasinoff, B. B., Wu, X., Krokhin, O. V., Ens, W., Standing, K. G., Nitiss, J. L., et al. (2005). Biochemical and proteomics approaches to characterize topoisomerase IIalpha cysteines and DNA as targets responsible for cisplatininduced inhibition of topoisomerase IIalpha. Mol. Pharmacol. 67, 937-947. doi: 10.1124/mol.104.004416

Hasinoff, B. B., Wu, X., and Yang, Y. (2004). Synthesis and characterization of the biological activity of the cisplatin analogs, cis- $\mathrm{PtCl} 2$ (dexrazoxane) and cis-PtCl2(levrazoxane), of the topoisomerase II inhibitors dexrazoxane (ICRF-187) and levrazoxane (ICRF-186). J. Inorg. Biochem. 98, 616-624. doi: 10.1016/j.jinorgbio.2004.01.008

Hernández-Martínez, S., Barradas-Bautista, D., and Rodríguez, M. H. (2013). Diferential DNA Synthesis In Anopheles Albimanus tissues induced by immune challenge with different microorganisms. Arch. Insect Biochem. Physiol. 84, 1-14. doi: 10.1002/arch.21108

Hernandez-Martinez, S., Román-Martínez, U., Martínez-Barnetche, J., Garrido, E., Rodríguez, M. H., and Lanz-Mendoza, H. (2006). Induction of DNA synthesis in Anopheles albimanus tissue cultures in response to a Saccharomyces cerevisiae challenge. Arch. Insect Biochem. Physiol. 63, 147-158. doi: 10.1002/arch.20150

Huang, W., Zhang, J., Yang, B., Beerntsen, B. T., Song, H., and Ling, E. (2016). DNA duplication is essential for the repair of gastrointestinal perforation in the insect midgut. Sci. Rep. 6:19142. doi: 10.1038/srep19142

Keller, O., Kollmar, M., Stanke, M., and Waack, S. (2011). A novel hybrid gene prediction method employing protein multiple sequence alignments. Bioinformatics 27, 757-763. doi: 10.1093/bioinformatics/btr010

Khoo, C. C., Doty, J. B., Held, N. L., Olson, K. E., and Franz, A. W. (2013). Isolation of midgut escape mutants of two American genotype dengue 2 viruses from Aedes aegypti. Virol. J. 10:257. doi: 10.1186/1743-422X-10-257

Lee, H. O., Davidson, J. M., and Duronio, R. J. (2010). Endoreplication: polyploidy with purpose. Genes Dev. 23, 2461-2477. doi: 10.1101/gad.1829209

Lefort, V., Longueville, J.-E., and Gascuel, O. (2017). SMS: smart model selection in PhyML. Mol. Biol. Evol. 34, 2422-2424. doi: 10.1093/molbev/msx149

Li, Y., Wu, S., Pu, J., Huang, X., and Zhang, P. (2015). Dengue virus up-regulates expression of notch ligands Dll1 and Dll4 through interferon- $\beta$ signalling pathway. Immunology 144, 127-138. doi: 10.1111/imm.12357

Ming, L., Wilk, R., Reed, B. H., and Lipshitz, H. D. (2013). Drosophila Hindsight and mammalian RREB-1 are evolutionarily conserved DNA-binding transcriptional attenuators. Differentiation 86, 159-170. doi: 10.1016/j.diff.2013.12.001

Moreno-García, M., Vargas, V., Ramírez-Bello, I., Hernández-Martínez, G., and Lanz-Mendoza, H. (2015). Bacterial exposure at the larval stage induced sexual immune dimorphism and priming in adult Aedes aegypti mosquitoes. PLoS ONE 10:e0133240. doi: 10.1371/journal.pone.0133240

Morens, D. M., Halstead, S. B., Repik, P. M., Putvatana, R., and Raybourne, N. (1985). Simplified plaque reduction neutralization assay for dengue viruses by semimicro methods in BHK-21 cells: comparison of the BHK suspension test with standard plaque reduction neutralization. J. Clin. Microbiol. 22, 250-254. 
OMS (2009). Dengue Guias Para El Diagnostico, Tratamiento, Prevencion Y Control. Oms Programa Especial de Investigaciones y Enseñanzas sobre Enfermedades Tropicales. 113-115.

Owczarzy, R., Tataurov, A. V., Wu, Y., Manthey, J. A., McQuisten, K. A., Almabrazi, H. G., et al. (2008). IDT SciTools: a suite for analysis and design of nucleic acid oligomers. Nucleic Acids Res. 36, W163-W169. doi: 10.1093/nar/gkn198

Palmer, W. H., Jia, D., and Deng, W.-M. (2014). Cis-interactions between Notch and its ligands block ligand-independent Notch activity. Elife 3:e04415. doi: 10.7554/eLife.04415

Pickup, A. T., Ming, L., and Lipshitz, H. D. (2009). Hindsight modulates Delta expression during Drosophila cone cell induction. Development 136, 975-982. doi: $10.1242 /$ dev.027318

Ramirez, J. L., Souza-Neto, J., Torres Cosme, R., Rovira, J., Ortiz, A., Pascale, J. M., et al. (2012). Reciprocal Tripartite Interactions between the Aedes aegypti Midgut Microbiota, Innate Immune System and Dengue Virus Influences Vector Competence. PLoS Negl. Trop. Dis. 6:e1561. doi: 10.1371/journal.pntd.0001561

Ramos-Castañeda, J., González, C., Jiménez, M. A., Duran, J., HernándezMartínez, S., Rodríguez, M. H., et al. (2008). Effect of nitric oxide on Dengue virus replication in Aedes aegypti and Anopheles albimanus. Intervirology 51, 335-341. doi: 10.1159/000175639

Raquin, V., and Lambrechts, L. (2017). Dengue virus replicates and accumulates in Aedes aegypti salivary glands. Virology 507, 75-81. doi: 10.1016/j.virol.2017.04.009

Romoser, W. S., Wasieloski, L. P., Pushko, P., Kondig, J. P., Lerdthusnee, K., Neira, M., et al. (2004). Evidence for arbovirus dissemination conduits from the mosquito (Diptera: Culicidae) midgut. J. Med. Entomol. 41, 467-475. doi: 10.1603/0022-2585-41.3.467

Salamov, A. A., and Solovyev, V. V. (2000). Ab initio gene finding in Drosophila genomic DNA. Genome Res. 10, 516-522. doi: 10.1101/gr.10.4.516

Salazar, M. I., Richardson, J. H., Sánchez-Vargas, I., Olson, K. E., and Beaty, B. J. (2007). Dengue virus type 2: replication and tropisms in orally infected Aedes aegypti mosquitoes. BMC Microbiol. 7:9. doi: 10.1186/1471-2180-7-9

Sánchez-Vargas, I., Scott, J. C., Poole-Smith, B. K., Franz, A. W. E., BarbosaSolomieu, V., Wilusz, J., et al. (2009). Dengue virus type 2 infections of Aedes aegypti are modulated by the mosquito's RNA interference pathway. PLoS Pathog. 5:1000299. doi: 10.1371/journal.ppat.1000299

Sim, S., and Dimopoulos, G. (2010). Dengue virus inhibits immune responses in Aedes aegypti cells. PLoS ONE 5:10378. doi: 10.1371/journal.pone.0010678

Slater, G., Birney, E., Box, G., Smith, T., Waterman, M., Altschul, S., et al. (2005). Automated generation of heuristics for biological sequence comparison. BMC Bioinformatics 6:31. doi: 10.1186/1471-2105-6-31

Souza-Neto, J. A., Sim, S., and Dimopoulos, G. (2009). An evolutionary conserved function of the JAK-STAT pathway in anti-dengue defense. Proc. Natl. Acad. Sci. U.S.A. 106, 17841-17846. doi: 10.1073/pnas.0905006106
Sun, J., and Deng, W.-M. (2007). Hindsight mediates the role of notch in suppressing hedgehog signaling and cell proliferation. Dev. Cell 12, 431-442. doi: 10.1016/j.devcel.2007.02.003

Terriente-Felix, A., Li, J., Collins, S., Mulligan, A., Reekie, I., Bernard, F., et al. (2013). Notch cooperates with Lozenge/Runx to lock haemocytes into a differentiation programme. Development 140, 926-937. doi: 10.1242/dev.086785

Tsujimoto, H., Hanley, K. A., Sundararajan, A., Devitt, N. P., Schilkey, F. D., and Hansen, I. A. (2017). Dengue virus serotype 2 infection alters midgut and carcass gene expression in the Asian tiger mosquito, Aedes albopictus. PLoS ONE 12:e0171345. doi: 10.1371/journal.pone.0171345

Vargas, V., Moreno-García, M., Duarte-Elguea, E., and Lanz-Mendoza, H. (2016). Limited specificity in the injury and infection priming against bacteria in Aedes aegypti mosquitoes. Front. Microbiol. 7:975. doi: 10.3389/fmicb.2016.00975

Xi, Z., Ramirez, J. L., and Dimopoulos, G. (2008). The Aedes aegypti toll pathway controls dengue virus infection. PLoS Pathog 4:e1000098. doi: 10.1371/journal.ppat.1000098

Ye, J., Coulouris, G., Zaretskaya, I., Cutcutache, I., Rozen, S., and Madden, T. L. (2012). Primer-BLAST: a tool to design target-specific primers for polymerase chain reaction. BMC Bioinformatics 13:134. doi: 10.1186/1471-210513-134

Yip, M. L., Lamka, M. L., and Lipshitz, H. D. (1997). Control of germ-band retraction in Drosophila by the zinc-finger protein HINDSIGHT. Development 124, 2129-2141.

Zanchi, C., Troussard, J.-P., Martinaud, G., Moreau, J., and Moret, Y. (2011). Differential expression and costs between maternally and paternally derived immune priming for offspring in an insect. J. Anim. Ecol. 80, 1174-1183. doi: 10.1111/j.1365-2656.2011.01872.x

Zdobnov, E. M., Tegenfeldt, F., Kuznetsov, D., Waterhouse, R. M., Simão, F. A., Ioannidis, P., et al. (2017). OrthoDB v9.1: cataloging evolutionary and functional annotations for animal, fungal, plant, archaeal, bacterial and viral orthologs. Nucleic Acids Res. 45, D744-D749. doi: 10.1093/nar/ gkw1119

Conflict of Interest Statement: The authors declare that the research was conducted in the absence of any commercial or financial relationships that could be construed as a potential conflict of interest.

Copyright (c) 2018 Serrato-Salas, Hernández-Martínez, Martínez-Barnetche, Condé, Alvarado-Delgado, Zumaya-Estrada and Lanz-Mendoza. This is an open-access article distributed under the terms of the Creative Commons Attribution License (CC $B Y)$. The use, distribution or reproduction in other forums is permitted, provided the original author(s) and the copyright owner are credited and that the original publication in this journal is cited, in accordance with accepted academic practice. No use, distribution or reproduction is permitted which does not comply with these terms. 